\title{
Remoción de arsénico presente en el agua potable del municipio de Ojocaliente, Zac. empleando perlas de gel de quitosano
}

\section{Removal of arsenic present in the drinking water of the municipality of Ojocaliente, Zac. using chitosan gel beads}

SANDOVAL-ALVARADO, Martha Verónicat’, CONEJO-FLORES, Ricardo’, GUZMÁNPANTOJA, Javier"“ y GARCÍA-GONZÁLEZ, Juan Manuel*'

\begin{abstract}
Universidad Autónoma de Zacatecas. Unidad Académica de Ciencias Químicas, Programa Académico de Ingeniería Química, Campus UAZ Siglo XXI. Carretera Zacatecas - Guadalajara Km. 6, Ejido la Escondida, 98160 Zacatecas, Zac. México.

"Gerencia de Refinación de Hidrocarburos, Instituto Mexicano del Petróleo, Eje Central Lázaro Cárdenas 152 Ciudad de México, 07730, México.
\end{abstract}

ID $1^{\text {er }}$ Autor: Martha Verónica, Sandoval-Alvarado / ORC ID: 0000-0002-6349-2036

ID $1^{\text {er }}$ Coautor: Ricardo, Conejo-Flores / ORC ID: 0000-0002-8513-1821

ID $2^{\text {do }}$ Coautor: Javier, Guzmán-Pantoja / ORC ID: 0000-0003-2103-3488

ID $3^{\text {er }}$ Coautor: Juan Manuel, García-González / ORC ID: 0000-0001-7259-5021

DOI: $10.35429 / J U S D .2020 .18 .6 .18 .27$

Recibido: 20 de Enero, 2020; Aceptado 30 de Junio, 2020

\section{Resumen}

Existe una gran problemática para el abastecimiento de agua potable en México, el estado de Zacatecas no es la excepción, aunado a esto el agua en la región se caracteriza por la presencia de metales pesados, compuestos de flúor, arsénico y alta dureza. El objetivo de este trabajo es remover el arsénico presente en el agua potable del municipio de Ojocaliente, Zac.; mediante el empleo de perlas de gel de quitosano. Se caracterizó la muestra inicial dando una concentración de As de $70 \mathrm{ppb}$, valor alto en referencia a la Norma Oficial Mexicana NOM-127-SSA1-1994 para el agua de consumo humano. Se realizaron una serie de experimentos a diferentes concentraciones, utilizando agua potable muestreada en Ojocaliente, Zac., la operación fue por lotes a una temperatura promedio de $19{ }^{\circ} \mathrm{C}$ y a $250 \mathrm{rpm}$, en alícuotas de $50 \mathrm{ml} \mathrm{y} 0.3 \mathrm{~g}$ de perlas de gel de quitosano. Se determinó el modelo de isoterma y de cinética que ajustan los datos, se obtiene que la isoterma de Langmuir y el de pseudo primer orden, respectivamente, y se removió en una etapa de separación el $20 \%$ del arsénico presente en el agua.

\begin{abstract}
There is a great problem for the supply of drinking water in Mexico, the state of Zacatecas is no exception, in addition to this the water in the region has the presence of heavy metals, fluorine compounds, arsenic and high hardness. The objective of this work is to remove the arsenic present in the drinking water of the municipality of Ojocaliente, Zac. using chitosan gel beads. The initial sample was characterized giving an As concentration of $70 \mathrm{ppb}$, a high value in reference to the Official Mexican Standard NOM-127-SSA1-1994 for water for human consumption. A series of experiments were carried out at different concentrations, using drinking water sampled in Ojocaliente, Zac., The operation was in batches at an average temperature of $19^{\circ} \mathrm{C}$ and $250 \mathrm{rpm}$, in aliquots of $50 \mathrm{ml}$ and $0.3 \mathrm{~g}$ of gel chitosan beads. The isotherm and kinetics model that fit the data was determined, being Langmuir and Pseudo first order, respectively, and 20\% of the arsenic present in the water was removed in a separation step.
\end{abstract}




\section{Introducción}

Existe una gran problemática para el abastecimiento de agua potable en México, el estado de Zacatecas no es la excepción. La Junta Local de Agua Potable y Alcantarillado de cada uno de los municipios se enfrenta al tipo de agua que deben de tratar para suministrar su servicio. De acuerdo a Camacho (2017), este tipo de organismos tienen la responsabilidad de proveer los servicios de agua potable y saneamiento a los ciudadanos y la forma en que estas dependencias públicas funcionan, incide directamente en la salud, seguridad y vida digna de las personas (Camacho, 2017). La ciudad de Ojocaliente, Zacatecas se encuentra ubicada con Latitud Norte de $22^{\circ} 34^{\prime} 19^{\prime \prime}$ Longitud Oeste de $102^{\circ}$ $15^{\prime} 10^{\prime \prime}$, con una altura sobre el nivel del mar de $2045 \mathrm{~m}$. Su territorio es de $645 \mathrm{~m}^{2}$. Con una población de 22278 mujeres y 21193 hombres (INEGI, 2017). Con una temperatura promedio anual de $15.9^{\circ} \mathrm{C}$ y una precipitación promedio anual de 395.5 (Medina, 2004). A la ciudad de Ojocaliente, Zacatecas, toma el agua del Acuífero del mismo nombre definido con la clave 3212 en el Sistema de Información Geográfica para el Manejo de Aguas Subterráneas (SIGMAS) de la CONAGUA, se localiza en la porción suroriental del estado de Zacatecas, en el límite con el estado de Aguascalientes; entre los paralelos $22^{\circ} 17^{\prime}$ y $22^{\circ}$ $44^{\prime}$ de latitud norte y entre los meridianos $102^{\circ} 06^{\prime}$ y $102^{\circ} 38^{\prime}$ de longitud oeste, cubriendo una superficie aproximada de $1,627 \mathrm{~km}^{2}$. La concentración de sólidos totales disueltos (STD) varían de 256 a $780 \mathrm{mg} / \mathrm{l}$, que no supera el límite máximo permisible para el agua destinada al consumo humano. De acuerdo con la clasificación establecida por la American Public Health Association, el agua del acuífero se clasifica como agua dulce, ya que los valores de conductividad eléctrica son inferiores a 2000 $\mu \mathrm{S} / \mathrm{cm}$ y presentan valores extremos de 175 a $900 \mu \mathrm{S} / \mathrm{cm}$. Las temperaturas registradas variaron de 24.4 a $33.0{ }^{\circ} \mathrm{C}$ en tanto que el $\mathrm{pH}$ medido fluctuaba entre 5.6 a 7.8. De acuerdo a las concentraciones de elementos mayores por ion dominante, se clasificaron las muestras en el diagrama de Piper y se identificó que la familia del agua que predomina es la bicarbonatada-sódica, que representa agua de reciente infiltración con periodos cortos de residencia, que ha circulado a través de rocas volcánicas (Última caracterización realizada en 1977) (CONAGUA, 2018).
El objetivo de este trabajo es remover el arsénico presente en el agua potable del municipio de Ojocaliente, Zac., bajo el empleo de perlas de gel de quitosano.

De acuerdo con la información en la literatura, los estudios y trabajos que se han realizado para solucionar el problema del arsénico en el agua es muy amplio. Se pueden encontrar desde procesos físicos, químicos o fisicoquímicos. El proceso más empleado es el de la adsorción como ejemplo de ello se tiene los trabajos realizados por: Petkova (1999), en el cual utilizó la hematita para el tratamiento de muestras de agua del Estado de Hidalgo, con una disminución en la concentración a valores de 0.05 mg/L de arsénico (Petkova S. V., 1999). Rivera et al (2002), emplearon dos plantas potabilizadoras piloto, con capacidad de $0.8 \mathrm{l} / \mathrm{s}$, mediante adsorción con alúmina activada, para remover el arsénico pentavalente y con la consideración de un paso intermedio para la transformación del arsénico trivalente a pentavalente. La eficiencia de remoción del arsénico con el tren de tratamiento fue del 90 al 100\% (Rivera H. M., 2002). Iliná et al (2009), emplearon la cáscara y su fibra de maracuyá para la remoción de arsenatos y arsenitos de agua preparada sintéticamente (Iliná, 2009). Alarcón (2010), en su trabajo presenta los resultados obtenidos en la remoción de arsénico en agua utilizando películas de TiZnOy:Fe. Removió el $80 \%$ del arsénico presente (Alarcón A. , 2010).

Montero et al (2010), utilizaron quitosano para remover el arsénico del agua, retirando el $87 \%$ (Montero, 2010). Pelizzari (2015), realizaron la degradación de arsénico empleando Pseudomonas aeruginosa para bioremediación de agua subterránea, con una remoción del 60 \% (Pellizzari, 2015). Meichtry et al (2015) removieron arsénico del agua mediante adsorción y/o co-precipitación bajo el empleo de materiales económicos como arcilla natural y hierro cerovalente comercial, microparticulado y como viruta (Meichtry, 2015).

Starbird y Montero (2015), reportan que las nanoparticulas de de fierro con propiedades supermagnéticas elaborado por ellos presentan adecuadas propiedades para ser utilizado en el tratamiento de agua de consumo humano (Starbird, 2015). 
Serafín et al (2016), emplearon pellets, provenientes de residuos lignocelulósicos, para el tratamiento de aguas contaminadas con arsénico, removiendo el $98.50 \% \pm 1.2 \%$ (Serafín, 2016). Liñan (2016), determinó la capacidad que presentan las raíces de cebolla, Allium cepa para remover arsénico del agua, encontrando que remueven más del $50 \%$ (Liñan, 2016). Berardozzi et al (2017), removieron arsénico de agua subterránea mediante un prototipo que usa adsorción y/o coprecipitación empleando la técnica de Hierro Cero Valente (ZVI), encontrando que mediante esta técnica se puede remover contenidos de arsénico del orden de 100 a 150 ppb (Berardozzi, 2017). Rojas (2017), presenta la posibilidad de aplicar a escala de laboratorio una técnica que involucra dos medios adsorbentes, óxidos de hierro y carbón activado producido artesanalmente a base de cáscara de jícaro sabanero (Crescentia alata), removiendo $50 \%$ y $58 \%$ respectivamente (Rojas C. B., 2017). López et al (2017), emplean silicato de sodio bajo condiciones de hidrólisis ácida y como agente sililante el 3mercaptopropiltrimetoxisilano bajo las mismas condiciones. Se estudió la capacidad adsorbente del material obtenido para la remoción de arsénico de soluciones acuosas, no especifican el porcentaje de remoción (López, 2017). Santos et al (2017), analizaron la capacidad de remoción de arsénico (As V) en solución acuosa por la biomasa modificada del hongo Aspergillus niger, removiendo el $96 \%$ del As (Santos, 2017).

Martínez (2018), uso un compósito preparado a partir de carbón activado granular y una solución de desecho proveniente de un proceso hidrometalúrgico con un alto contenido de hierro, se logró reducir una concentración inicial de $2000 \mu \mathrm{g} / \mathrm{L}$ a valores por debajo de 10 $\mu \mathrm{g} / \mathrm{L}$ de As(V) (Martínez R. M., 2018). Piña et al (2018), instalaron tres sistemas de lecho fijo para evaluar la capacidad de adsorción del arsénico sobre el material adsorbente NXT®-2, cumplieron con el límite de la NOM-127 de 25 $\mu \mathrm{g} / \mathrm{L}$ en el efluente (Piña, 2018). Martínez et al (2018), demostraron que la adsorción de arsénico requiere de adsorbentes eficientes y económicos, por lo cual los óxidos de hierro, tanto naturales como sintéticos, son los adsorbentes más utilizados para eliminar arsénico del medio acuoso, debido a su alta capacidad de adsorción, accesibilidad y bajo costo (Martínez L. M., 2018).
Rivera y Piña (sf), usaron como medio adsorbente zeolita natural (clinoptilolita) recubierta con óxidos de hierro, para remover arsénico de aguas naturales, obteniendo una concentración final inferior al límite máximo permisible $(0.045 \mathrm{mg} / \mathrm{l})$ establecido en la modificación a la norma NOM127-SSA1-1994 (Rivera H. M., sf). Petkova et al (sf), realizaron pruebas en lotes con 17 minerales entre ellos zeolita tipo clinoptilolita, carbón activado, arena $\mathrm{y}$ óxidos de hierro, manganeso $\mathrm{y}$ aluminio.

Los minerales con los que se obtuvieron las mayores remociones fueron utilizados en pruebas en continuo. En esta última etapa se lograron remociones hasta del $100 \%$ con alúmina activada, 97\% con hematita, 99\% con arena recubierta con óxidos de hierro y $96 \%$ con zeolita recubierta con óxidos de manganeso (Petkova S. V., sf). Rojas y Sánchez (2020), utilizaron Bentonita como material adsorbente, añadiéndole Óxido de Grafeno (GO) y evaluaron la capacidad de adsorción del material compuesto, concluyendo que se remueve el Arsénico en un porcentaje mayor al 95\% (Rojas G. D., 2020).

Martínez et al (2020), demostraron que, el material magnético nanoestructurado recubierto con quitosano fúngico remueve el $100 \%$ del arsénico presente en muestras de agua proveniente de un pozo de la Comarca Lagunera (Martínez P. V., 2020). Otra forma de remover el arsénico propuesta en la literatura es mediante la filtración, los trabajos relevantes son: Calderón et al (2012), evaluaron la viabilidad técnica y económica de potabilizar agua contaminada con arsénico mediante procesos con membranas, demostrando que se tiene valores más bajos que los límites superiores dictados por la Norma para la presencia de arsénico en el agua potable (Calderón, 2012). Escalera (2016), evaluaron el desempeño de un sistema de filtración para la remoción simultánea de $\mathrm{As}$ y $\mathrm{Fe}$, mediante filtros de propileno. Las eficacias de remoción de As total y Fe total en el sistema fueron significativas $\quad(33-41 \% \quad y \quad 46-78 \%$, respectivamente) (Escalera V. R., 2016). 
Villa et al (2018), usaron un filtro tradicional de arena y un filtro con el material adsorbente de hierro cerovalente estabilizado en quitosano, a partir de una concentración promedio de $0,51 \mathrm{mg} / \mathrm{L}$, se redujo la concentración de arsénico en el rango de 0,005 y 0,025 mg/L (Villa, 2018). González et al (sf), alcanzaron una eficiencia de remoción de arsénico fueron del 97 al 98\%, mediante filtración directa con hierro como coadyuvante (González, sf). Siguiendo con el análisis de procesos para la remoción de arsénico se tiene los referentes a la coagulación, que a continuación se presentan: Castruita et al (2011), en su trabajo presenta la remoción de arsénico de agua natural y preparada exprofeso para la experimentación en un reactor electroquímico tipo filtro prensa (Castruita, 2011).

\begin{tabular}{cccc}
\multicolumn{2}{c}{ Francisca et al (2014), realizaron } \\
procesos de
\end{tabular} obtuvieron porcentajes de remediación de arsénico que variaron desde 0 hasta $95 \%$ (Francisca \& Carro, 2014). Araya et al (2016), obtuvieron eficiencias de remoción cercanas al $96 \%$, obteniendo concentraciones de arsénico en el efluente menores a $10 \mu \mathrm{g} / \mathrm{L}$, empleando filtración rápida con arena y cloruro de hierro (III) junto con la adición de dos floculantes, uno sintético (polímero catiónico KF-930-S) y otro natural (mozote Triumfetta semitriloba Jacq, Malvaceae) (Araya, 2016). Aguirre (2015), empleó el sulfato férrico como coagulante, removió hasta el $97 \%$ del arsénico de $60 \mu \mathrm{g} / \mathrm{L}$ en agua cruda hasta $2 \mu \mathrm{g} / \mathrm{L}$ en agua decantada (Aguirre, 2015). Paitan y Palomino (2017), removieron el arsénico del agua potable por el método de electrocoagulación, retirando el $97.73 \%$ (Paitan, 2017). Garrido (2019), optimizó los procesos de espesamiento, acondicionamiento y deshidratación de los residuos generados en la coagulaciónfloculación-sedimentación y filtración directa de agua que contienen altas concentraciones de arsénico, alcanzando los límites permisibles (Garrido, 2019).

Otro de los métodos de remoción son los de óxido reducción que a continuación se presentan los trabajos encontrados en la literatura: Litter et. al. (2003), en su trabajo presentaron los estudios preliminares de adaptación y validación de la tecnología de Remoción de Arsénico por Oxidación Solar (RAOS).
Mediante esta tecnología aplicada en Los Pereyra, Provincia de Tucumán, Argentina se logró la remoción de más del $90 \%$ de arsénico presente. De igual forma, al emplearla en las aguas del Rio Camarones, Arica, Chile, se removió el $70 \%$ del arsénico presente. Por último en zonas rurales Lima, Perú se logró el $95 \%$ de remoción con la tecnología RAOS (Litter M. M., 2003). Chávez y Miglio (2011), removieron el $88 \%$ de arsénico presente en el agua del rio Iscahuaca-Colcabamba utilizando el método de Remoción de Arsénico por Oxidación Solar, alambre del No. 16 y jugo de limón de la variedad sutil (Chávez \& Miglio, 2011).

Rojas et al (2015), presentan dos soluciones a nivel domiciliar para eliminar el arsénico: una por oxidación solar (SORAS) y otra por un proceso de coagulación-floculación. En el sistema SORAS se encontró que para reducir la concentración de arsénico de $200 \mu \mathrm{g} / \mathrm{L}$ a menos de la Norma $(10 \mu \mathrm{g} / \mathrm{L})$, se necesitan entre $2 \mathrm{~h}$ y $4 \mathrm{~h}$ de exposición en un día soleado o nublado, respectivamente. (Rojas C. P., 2015). Carranza (2015), evaluó dos tecnologías artesanales para la remoción de plomo y arsénico en agua para consumo humano: la unidad de tratamiento con dos cubetas y el método de remoción asistido por luz solar (RAOS), para la remoción del plomo fueron: por el método RAOS el porcentaje de remoción fue de $99.98 \%$ y para la Unidad de tratamiento con dos cubetas fue del $99.92 \%$. Para el caso del arsénico el porcentaje de remoción por el método RAOS es del $81.5 \%$ y para la Unidad de tratamiento con dos cubetas fue de $83.5 \%$ (Carranza, 2015). Chapa (2018), en su trabajo reduce el arsénico hasta un $99.8 \%$ al aplicar la técnica de oxidación solar (Chapa, 2018).

Otros estudios que complementan son los realizados por: Pérez et al (2003), muestran en su estudio el trabajo cooperativo desarrollado en América Latina respecto a la evaluación y manejo del riesgo de exposición a arsénico en el agua potable, así como, los métodos principales para la remoción de arsénico. Un ejemplo presentado es el uso de coagulantes (Sulfato de alúmina) que alcanza una remoción del arsenito igual a $50 \%$ y del arsenato un $90 \%$ (Pérez, 2003). 
Litter (2006), en su Informe Final del Proyecto OEA/AE141 presentan los principales resultados en países del Tercer Mundo al aplicar la tecnología solar para la potabilización del agua (Litter M. , 2006).

Alarcón et al (2012), en su estudio presentan la presencia de arsénico en el agua de consumo humano en Latinoamérica, los niveles máximos permisibles considerados, técnicas de medición, los procesos convencionales de remoción, así como las nuevas tecnologías en desarrollo (Alarcón H. L., 2012). Escalera y Ormachea (2017), en su estudio evaluaron la viabilidad técnica de la mayoría los procesos de remoción de arsénico: coagulación con $\mathrm{Fe}(\mathrm{III})$ y Al(III), adsorción sobre alúmina activada, intercambio iónico, filtración en lechos de arena recubierta con óxido de hierro (IHE-ADART), Remoción de Arsénico Asistida por Oxidación Solar, RAOS, nano-filtración y osmosis reversa, concluyendo que pueden ser empleados para tal fin, lo que determinara cual es el que puede ser empleado lo dictará la viabilidad económica (Escalera V. R., 2017).

Escarcena (2018), remueve el arsénico mediante precipitación alcalina, alcanzando a retirar el $78 \%$ promedio (Escarcena, 2018). Torres et al (2020), propusieron el uso de biomembranas compuestas con residuos agroindustriales que presentan diversas ventajas para filtrar el agua y eliminar eficientemente el As (Torres, Manjarrez, \& Vega, 2020). Rojas y Suyon (2020), evaluaron la eficiencia de fitorremediación con Jacinto de agua (Eichhornia crassipes) para la disminución de las concentraciones de arsénico en aguas del centro poblado Cruz del Médano, alcanzando a disminuir el arsénico en un $60 \%$ del inicial (Rojas \& Suyon, 2020).

La relevancia de este estudio es que solo se han realizado dos investigaciones empleando quitosano y no en forma de perlas, como aquí se realizó.

\section{El Quitosano}

Es un producto derivado de la quitina, es un biopolímero tiene toxicidad nula y es biodegradable. Está constituido por unidades repetidas de D-glucosamina, por lo que a longitud de la cadena también es tomada como una característica importante (Figura 1).
El quitosano se conoce también por ser soluble en algunas soluciones acuosas de varios ácidos. Este material tiene al igual que la quitina diversos usos para distintas industrias.

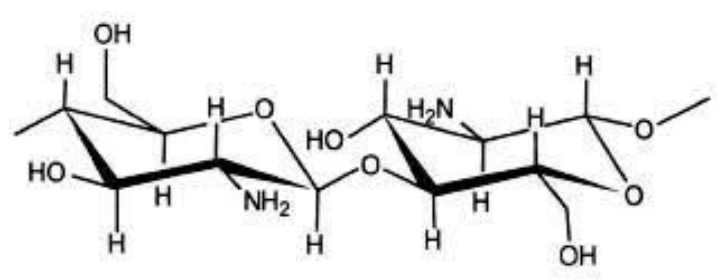

Figura 1 Estructura del quitosano

Fuente: (Conejo, 2012)

Este polímero se caracteriza por tener un elevado número de grupos amino libres que son muy reactivos para la quelación de iones. Las propiedades del quitosano conducen a la protonación de los grupos amino a $\mathrm{pH}$ ácido; estas propiedades catiónicas hacen que el polímero sea muy eficiente en la adsorción de iones.

\section{La adsorción}

La adsorción es el proceso en el cual se realiza la captación de sustancias solubles presentes en una solución por una interfase conveniente. La interfase puede encontrarse entre el líquido y un gas, un sólido u otro líquido (Metcalf \& Eddy, 2003), Una medida de la posición de equilibrio en el proceso de adsorción está en función de la concentración del soluto, naturaleza de los solutos competitivos, concentración, naturaleza de la solución.

La forma preferida de expresar esta distribución consiste en expresar la cantidad de soluto adsorbido por unidad de peso de adsorbente sólido como una función de la concentración del soluto que permanece en solución en equilibrio; a una temperatura fija. Una expresión de este tipo son las isotermas de adsorción (Weber, 2003). La variación de la cantidad de adsorción por unidad de masa con la concentración puede representarse por los modelos desarrollados por Langmuir, por Freundlich, por Brunauer, Emmet y Teller (BET), Temkin, Dubinin-Radushkevich y otros. Son las expresiones matemáticas más empleadas para describir los datos experimentales de la isoterma (Figura 2) (Félez, 2009). 


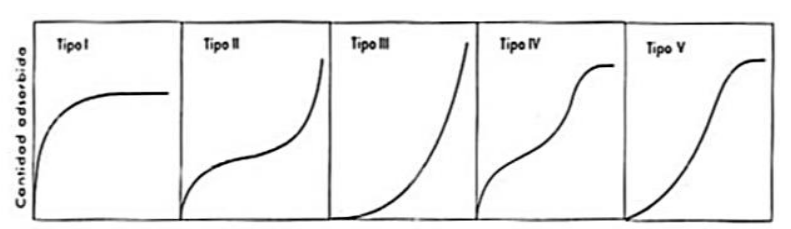

Figura 2 Tipos de isotermas de adsorción Fuente: (Medina, 2005)

El modelo teórico más simple para la adsorción en monocapa es el de Langmuir (Douglas, 1984)

$\frac{M_{A}}{M_{0}}=\frac{K C_{A}}{1+K C_{A}}$

Donde; $\mathrm{C}_{\mathrm{A}}$ es la concentración de $\mathrm{A}$ en la fase fluida, $\mathrm{M}_{0}$ es la concentración máxima de $\mathrm{A}$ retenido por el adsorbente, $\mathrm{M}_{\mathrm{A}}$ es la concentración de A retenido por el adsorbente y $\mathrm{K}$ es la constante de adsorción.

Con lo que respecta a la cinética de la adsorción y principalmente de metales, es influenciada por las reacciones de sorción y los pasos de transferencia de masa que gobiernan la transmisión de iones metálicos de la masa de la solución a los sitios activos en la superficie del sorbente, es decir, la difusión externa e interna. Para identificar el paso controlante en el mecanismo de la cinética de la adsorción se pueden considerar tres pasos principales:

1. Transferencia de masa de los iones metálicos desde la solución a la superficie del adsorbente.

2. Sorción de los iones metálicos en los sitios activos.

3. Difusión interna del ion en la partícula adsorbente.

Para el análisis de la cinética se han utilizado variados modelos simplificados; como lo son: la ecuación de pseudo-primer orden, ecuación de pseudo-segundo orden y difusión intrapartícula. El modelo cinético de pseudo primer orden propuesto por Langergren (Langergren, 1898) es definido como:

$\ln \left(q_{e}-q_{t}\right)=\ln q_{e}-k_{1} t$

Donde; $k_{1}$ es la constante de velocidad de adsorción de pseudo-primer orden, $q_{t}$ es la cantidad adsorbida a tiempo $t, t$ es el tiempo $q_{e}$ es la cantidad adsorbida en el equilibrio.

\section{Metodología para desarrollar}

Con base en las Normas de CONAGUA, SEMARNAT y las Normas Oficiales Mexicanas; NOM-014-SSA1-1993 y NOM230-SSA1-2002 se muestreó el agua en diferentes puntos de la ciudad de Ojocaliente, Zac., se mezclaron las muestras para homogenizarla conjuntando un volumen de 20 L. Para el análisis fisicoquímico se tomó como referencia la Norma Oficial Mexicana NOM127-SSA1-1994 (límites permisibles para calidad y tratamientos a los que se debe someter el agua para el uso y consumo humano). Se determinaron: Dureza (Se empleó un equipo de HANNA-Instruments HI3812); $\mathrm{pH}$ y conductividad (se utilizó un equipo HANNAInstruments HI98129); cloruros (se utilizó un equipo HANNA-Instruments HI3815); fluoruros (un equipo HANNA-Instruments HI739); arsénico (un kit HACH 2800000). Para las pruebas de sorción del arsénico en perlas de gel de quitosano, se realizaron series de experimentos en lotes. Para el régimen por lotes se emplearon muestras de $50 \mathrm{ml}$ por triplicado, se varió la concentración del arsénico en el agua muestreada añadiendo agua desionizada, a cada matraz se le depositaron $0.3 \mathrm{~g}$ de perlas de gel quitosano, se agitó a $250 \mathrm{rpm}$ en una parrilla de agitación y a una temperatura de $19{ }^{\circ} \mathrm{C} \pm 0.5$ por $5 \mathrm{~h}$ (para determinar el modelo de adsorción), para la cinética por $2 \mathrm{~h}$.

\section{Resultados}

En la Tabla 1 se presenta la caracterización realizada a la muestra original del agua potable de la ciudad de Ojocaliente, Zac, así como la desviación estándar de los datos obtenidos.

\begin{tabular}{|l|r|}
\multicolumn{1}{|c|}{ Componente } & \multicolumn{1}{c|}{ Valor } \\
\hline Dureza $(\mathrm{ppm})$ & $136.8 \pm 32.77$ \\
\hline $\mathrm{pH}$ & $9.766 \pm 0.83$ \\
\hline Conductividad $(\mu \mathrm{s})$ & $0.414 \pm 0.09$ \\
\hline Cloruros $(\mathrm{ppm})$ & 30 \\
\hline Fluoruros $(\mathrm{ppm})$ & $3.56 \pm 0.05$ \\
\hline Arsénico $(\mathrm{ppb})$ & $40 \pm 5$ \\
\hline Temperatura $\left({ }^{\circ} \mathrm{C}\right)$ & $19.26 \pm 0.17$ \\
\hline
\end{tabular}

Tabla 1 Caracterización del agua potable de Ojocaliente, Zac.

En la Tabla 2 se presentan los resultados obtenidos de la sorción del arsénico en las perlas de gel de quitosano. 


\begin{tabular}{|c|c|c|}
\hline $\begin{array}{c}\text { Diluciones } \\
\text { Agua desionizada }+ \\
\text { muestra }(\mathrm{ml})\end{array}$ & $\begin{array}{c}\mathrm{C}_{0} \\
(\mathbf{p p b})\end{array}$ & $\begin{array}{c}q \\
\text { ( } \mu \mathrm{g} / \mathrm{g} \text { de perlas de } \\
\text { quitosano) }\end{array}$ \\
\hline $135+15$ & 7 & 0.83 \\
\hline $120+30$ & 14 & 1.17 \\
\hline $90+60$ & 28 & 2.00 \\
\hline $60+90$ & 42 & 2.33 \\
\hline $30+120$ & 56 & 2.33 \\
\hline $15+135$ & 63 & 2.33 \\
\hline
\end{tabular}

Tabla 2 Resultados de la sorción de arsénico en perlas de gel de quitosano

Buscando el modelo de isoterma a la cual se ajustan los datos se encontró que el modelo que mejor ajusta es el de Langmuir. Los parámetros del modelo son los siguientes: $\mathrm{q}_{\mathrm{m}}=$ $3.21806 \mu \mathrm{g} / \mathrm{g}$ de sorbente; $\mathrm{K}=0.051791$, con un coeficiente de Pearson de 0.9775 (determinado con Excel), lo que se puede interpretar que la adsorción se realiza en una monocapa (lo cual no se pudo comprobar al no haber caracterizado el quitosano después de las pruebas de sorción).

En el gráfico 1, se presentan los datos experimentales y el modelo de Isoterma de Langmuir. El porcentaje de error al hacer el comparativo de los datos experimentales con el modelo es de $6.17 \%$.

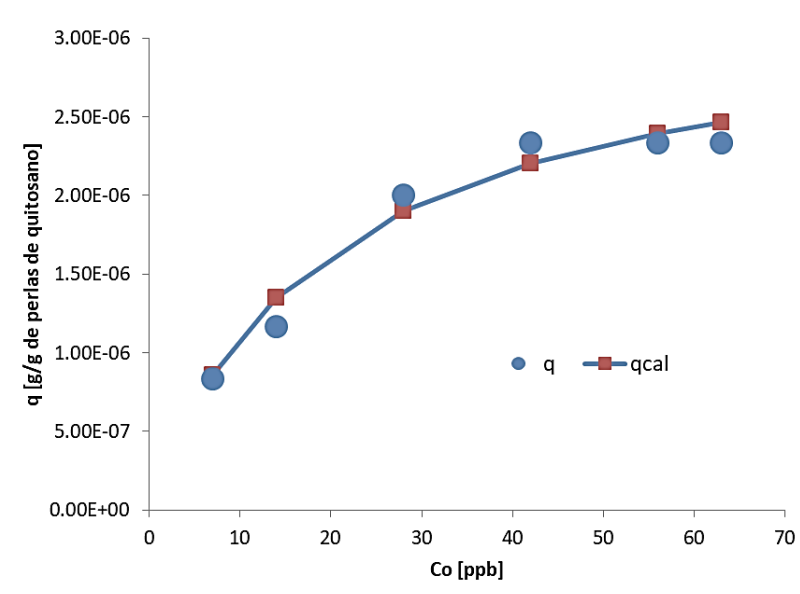

Gráfico 1 Datos experimentales (q) y el modelo de Langmuir ( $\left.q_{\text {cal }}\right)$

En la Tabla 3, se muestran los resultados al realizar las pruebas para determinar la cinética de adsorción.

\begin{tabular}{|r|r|}
\hline $\mathbf{t}$ [min] & $\mathbf{q}$ [ $\boldsymbol{\mu g}$ As/g de adsorbente] \\
\hline 0 & 0.00 \\
\hline 15 & 0.20 \\
\hline 30 & 1.17 \\
\hline 45 & 1.75 \\
\hline 60 & 2.04 \\
\hline 75 & 2.92 \\
\hline 90 & 2.92 \\
\hline 105 & 2.92 \\
\hline 120 & 3.79 \\
\hline
\end{tabular}

Tabla 3 Resultados para la cinética de adsorción del As en perlas de gel de quitosano

Los datos se ajustaron al modelo cinético de pseudo primer orden, los parámetros determinados para el modelo son: $\mathrm{q}_{\mathrm{e}}=4.34$ $\mu \mathrm{g} / \mathrm{g}$ de adsorbente y $k_{1}=-0.0124 \mathrm{~min}^{-1}$, con un coeficiente de Pearson de 0.9435 (Determinado con Excel). el porciento de error al ajustar los datos es de $11.21 \%$.

En el Gráfico 2, se presenta el ajuste de los datos al modelo de pseudo primer orden y los datos obtenidos en la experimentación.

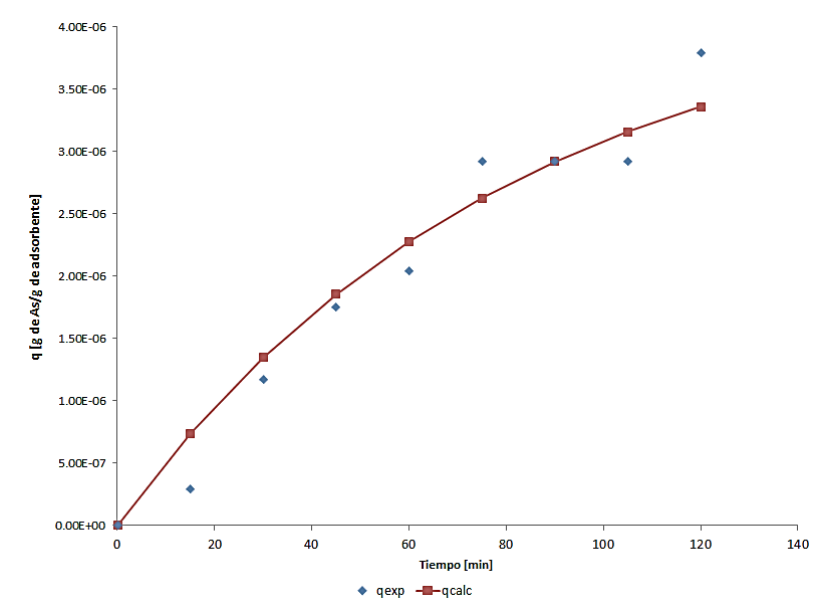

Gráfico 2 Datos experimentales ( $\mathrm{q}_{\exp }$ ) y el modelo cinético de pseudo primer orden $\left(\mathrm{q}_{\mathrm{cal}}\right)$

\section{Conclusiones}

Las perlas de gel de quitosano en una sola etapa de separación retiran de la muestra del agua potable el $27 \%$ del arsénico presente. Sería conveniente el evaluar en una serie de etapas para verificar si disminuye hasta los límites permisibles por la Norma.

Si se emplean los modelos de isoterma con los datos ajustados (Isoterma de Langmuir), y de igual forma, para la cinética de adsorción (Pseudo primer orden) se puede simular y optimizar un proceso más eficiente de separación. 
Aunque el quitosano es de bajo precio y biodegradable, es necesario realizar una evaluación económica para ser utilizado en el proceso de separación.

De los trabajos a futuro será determinar los ciclos de adsorción y desorción, para determinar el tiempo de operación de las perlas de gel de quitosano en este sistema.

\section{Referencias}

Aguirre, C. F. (2015). Efecto del proceso de coagulación con sulfato férrico en la remoción de arsénico del agua de Río Rímac, Aris Industrial SA, Lima-2014. Lima, Perú: Universidad Cesar Vallejo, Lima, Perú.

Alarcón, A. (2010). Películas delgadas de TiZnOy:Fe para remoción de arsénico en agua. Invest Apl Innov 4(1), 15 - 21.

Alarcón, H. L. (2012). Remoción de Arsénico en Agua de Consumo Humano en Latinoamérica. Cd de M'rxico: Centro de Investigación en Materiales Avanzados, S.C.

Araya, O. A. (2016). Remoción de arsénico en agua de consumo humano mediante la técnica de coagulación-floculación. Revista Ciencia, Tecnología y Salud Vol. 3 Num. 2 2016, 24093459.

Berardozzi, E. O. (2017). Planta piloto para la remoción de arsénico en agua: Prueba de campo . $4^{\circ}$ Jornadas ITE - 2017. La Plata: Facultad de Ingeniería - UNLP.

Calderón, M. Q. (2012). Remoción de arsénico mediante procesos de membrana. Tecnología y Ciencias del Agua, 37-51.

Camacho, H. C. (2017). Regulación de los servicios de agua potable y saneamiento en México. Jiutepec, Mor.: Instituto Mexicano de Tecnología del Agua .

Carranza, E. .. (2015). Evaluación de dos tecnologías artesanales para la remoción de plomo y arsénico en agua para consumo humano. . San Salvador: Universidad de El Salvador.

Castruita, Á. L. (2011). Estudio cinético de la remoción de arsénico en un reactor electroquímico tipo filtro prensa. Prospectiva, vol. 9, núm. 1, enero-junio, 7-12.
Chapa, R. L. (2018). Oxidación solar en la reducción de la concentración del arsénico en aguas del rio Rímac - cuenca alta, de la localidad San Mateo - Huarochirí, 2018. Lima, Perú: Universidad Cesar Vallejo.

Chávez, Q. M., \& Miglio, T. M. (2011). Remoción de arsénico por oxidación solar en aguas para consumo humano. Rev Soc Quím Perú. 77 (4), 307-314.

CONAGUA. (2018). Actualización de la disponibilidad media anual de agua en el acuifero Ojocaliente (3212), Estado de Zacatecas. cd. de México: Diario Oficial de la federación.

Escalera, V. R. (2016). Remoción de arsénico en aguas subterráneas con bajas concentraciones de hierro mediante microfiltros. comerciales. Investigación \& Desarrollo, No. 16, Vol. 1, 39-48.

Escalera, V. R. (2017). Hidroquímica de la presencia natural de arsénico en aguas subterráneas de áreas suburbanas de Cochabamba-Bolivia y evaluación de la viabilidad técnica de procesos de remoción. Investigación \& Desarrollo, No. 17, Vol. 1, 2741.

Escarcena, F. C. (2018). Remoción del arsénico de las aguas municipales y pozos domésticos en la ciudad de Juliaca por precipitación alcalina. Puno, Perú: Universidad Nacional del Altiplano.

Francisca, F. M., \& Carro, P. M. (2014). Remoción de arsénico en agua mediante procesos de coagulación-floculación. Int. Contam. Ambie. 30 (2), 177-190.

Garrido, H. S. (2019). Optimización de los procesos de tratamiento de residuos provenientes de la remoción de arsénico en agua para consumo humano. Cuernavaca, Mor.: IMTA.

González, P. R. (sf). Remoción de arsénico en agua de pozo profundo. $6^{\circ}$ Foro del Agua "El agua nos mueve a todos”. . Gluadalajara: U de G.

Iliná, A. M. (2009). Biosorción de arsénico en materiales derivados de maracuyá. Int. Contam. Ambient. 25 (4), 201-216.

SANDOVAL-ALVARADO, Martha Verónica, CONEJO-FLORES, Ricardo, GUZMÁN-PANTOJA, Javier y GARCÍA-GONZÁLEZ, Juan Manuel. Remoción de arsénico presente en el agua potable del municipio de Ojocaliente, Zac. empleando perlas de gel de quitosano. Revista del Desarrollo Urbano y Sustentable. 2020 
INEGI. (2017). Anuario estadístico y geográfico de Zacatecas 2017. Cd. de México: INEGI.

Liñan, A. R. (2016). Remoción de arsénico en agua por raíces de cebolla, Allium cepa, bajo condiciones de laboratorio. . Revista REBIOL. $36(2), 27-32$.

Litter, M. (2006). Resultados finales del Proyecto OEA/AE141: investigación, desarrollo, validación y aplicación de tecnologías solares para la potabilización de agua en zonas rurales aisladas de América Latina y el Caribe. Buenos Aires, Argentina: Agencia Interamericana para la Cooperación y Desarrollo.

Litter, M. M. (2003). Remoción de Arsénico Asistida por Luz Solar en Comunidades Rurales de América Latina. Buenos Aires, Argentina: Agencia Interamericana para la Cooperación y el Desarrollo.

López, P. C. (2017). Sílice Modificada con Grupos Mercapto por Reacción de Sililación para la Remoción de Arsénico en Agua. 52 Congreso Mexicano de Química y $36^{\circ}$ Congreso Nacional de Educación Química. Puerto Vallarta: Colección Memorias de los Congresos de la Sociedad Química de México.

Martínez, L. M. (2018). Remoción de arsénico de agua potable mediante adsorción sobre óxido y oxihidróxidos de hierro. Revista Ciencia Cierta No. 56., 1-14.

Martínez, P. V. (2020). Aprovechamiento de residuo de biomasa fúngica en la obtención de un sistema magnético nanoestructurado para remover arsénico del agua. Revista Internacional de Contaminación Ambiental, 36(2), , 383-397.

Martínez, R. M. (2018). Evaluación de un compósito adsorbente tipo carbón activadoóxido de hierro, como medio de remoción de arsénico de efluentes acuosos. . San Luís Potosí: Universidad Autónoma de San Luis Potosí.

Medina, G. G. (2004). ESTADÍSTICAS CLIMATOLÓGICAS BÁSICAS DEL ESTADO DE ZACATECAS (PERÍODO 1961-2003). Zacatecas: INIFAP.
Meichtry, J. M. (2015). Remoción de arsénico en agua mediante materiales de bajo costo y segura disposición final. Revista Proyecciones 13(2)., 47-58.

Montero, Á. J. (2010). Utilizaxión de quitosano para la remoción de arsénico (As) del agua. Revista Superficies y vacío, vol. 23, agosto, pp. 136-139. Sociedad Mexicana de Ciencia y Tecnología de Superficies y Materiales A.C. , 136-139.

Paitan, C. L. (2017). Remoción de arsénico del agua potable del Distrito De Quero Provincia de Jauja, por el método de electrocoagulación, a nivel laboratorio. Uancayo, Perú: Universidad Nacional del Centro del Perú.

Pellizzari, E. E. (2015). Degradación de arsénico por Pseudomonas aeruginosa para bioremediación de agua. estudio preliminar. Revista Avances en Ciencias e Ingeniería, vol. 6, núm. 1, enero-marzo, 1-6.

Pérez, F. C. (2003). Tecnología para el desarrollo humano agua e infraestructura. Madrid, España: Ingeniería sin Frontera.

Petkova, S. V. (1999). Estudio piloto para remoción del arsénico, Estado de Hidalgo, México. Ingeniería Hidráulica en México. Vol. XIV, No. 3, 65-77.

Petkova, S. V. (sf). Evaluación de diversos minerales para la remoción de arsénico de agua para consumo humano. Recuperado el 6 de 03 de 2020, de https://docplayer.es/22477533-Evaluacion-dediversos-minerales-para-la-remocion-dearsenico-de-aguapara-consumo-humano.html.

Piña, S. M. (2018). Evaluación TécnicoEconómica de un sistema para remoción de arsénico en agua para consumo humano. Cuernavaca, Mor.: IMTA.

Rivera, H. M. (2002). Remoción de hierro y arsénico de agua para consumo Humano mediante precipitación y adsorción en Zimapán,Hidalgo, México. XXVII Congresso Interamericano de Engenharia Sanitária $e$ Ambiental. ABES (págs. 1-8). Cancún, México: ABES. 
Rivera, H. M. (sf). Tratamiento de agua para remoción de arsénico mediante adsorción sobre zeolita natural acondicionada. . Recuperado el 6 de 03 de 2020, de https://www.scribd.com/document/335954923/ aguaarsenico2-pdf.

Rojas, A. L., \& Suyon, D. E. (2020). Eficiencia de fitorremediación con Jacinto de agua (Eichhornia crassipes) para disminuir concentraciones de arsénico en aguas del centro poblado Cruz del Medano - Morrope 2019. Chiclayo, Perú: Universidad de Lambayeque.

Rojas, C. B. (2017). Valoración de la eficiencia de carbón activado de jícaro sabanero (Crescentiaalata) y su combinación con óxidos de hierro para la remoción de arsénico en agua, 2016. Managua: Tesis de la Facultad de Ciencias e Ingeniería Departamento de Química Carrera: Química Ambiental. De la Universidad Nacional Autónoma De Nicaragua.

Rojas, C. P. (2015). Estudio de remoción de arsénico en agua potable a nivel domiciliar mediante oxidación solar y coagulaciónfloculación. Revista Tecnología en Marcha, Vol. 28, No. 4, Octubre-Diciembre, 55-65.

Rojas, G. D. (2020). Capacidad de adsorción de un material compuesto a base de bentonita y óxido de grafeno para remover arsénico en agua contaminada. Lambayeque, Perú.

Santos, D. E. (2017). Remoción de Arsénico (V) en Solución Acuosa por Biomasa Modificada del Hongo Aspergillus niger. Rev Información Tecnológica Vol. 28(6), 45-52.

Serafín, M. A. (2016). Uso potencial de pellets para el tratamiento de aguas contaminadas con arsénico en comunidades de Xichú, Gto., México. Revista Acta Universitaria, vol. 26, núm. 2, 22-32.

Starbird, P. R. (2015). Síntesis de nanopartículas magnéticas de óxido de hierro para la remoción de arsénico del agua de consumo humano. . Revista Tecnología en Marcha. Vol. 28, $N^{o}$ 3, Julio-Septiembre, 45-54.

Torres, C. C., Manjarrez, N. L., \& Vega, C. H. (2020). Separación de arsénico mediante biomembranas compósitas en agua de pozo del estado de Chihuahua. Glosa, Revista de Divulgación. Año 8. No 14,, 25-27.

ISSN 2414-4932

ECORFAN® Todos los derechos reservados
Villa, G. G. (2018). Evaluación de la remoción de arsénico en agua superficial utilizando filtros domiciliarios. Rev Peru Med Exp Salud Pública. 35(4), 652-656.
SANDOVAL-ALVARADO, Martha Verónica, CONEJO-FLORES, Ricardo, GUZMÁN-PANTOJA, Javier y GARCÍA-GONZÁLEZ, Juan Manuel. Remoción de arsénico presente en el agua potable del municipio de Ojocaliente, Zac. empleando perlas de gel de quitosano. Revista del Desarrollo Urbano y Sustentable. 2020 